\title{
Decision Making and Fuzzy Information
}

\author{
Owat Sunanta ${ }^{1 *}$, Reinhard Viertl ${ }^{2}$ \\ ${ }^{1}$ Department of Business and Management, Webster Vienna Private University, Vienna, Austria \\ ${ }^{2}$ Institute of Statistics and Mathematical Methods in Economics, Technische Universität Wien, Vienna, Austria \\ Email: owatsunanta43@webster.edu
}

\begin{abstract}
Information and its quality are essential for effective decision making, which often takes into account the statistical knowledge of the condition under consideration. Information in form of collected data from continuous quantities is always uncertain, i.e. more or less fuzzy. However, such data can be further described by so-called fuzzy numbers for better understanding. In applied statistics, a more general form of distributions (so-called fuzzy densities) is suitable to model uncertain stochastic information. The combination of fuzziness and stochastic uncertainty calls for a generalization of mathematical models for decision making. As a result, models must be generalized to handle this situation. This is possible and will be explained via decision model and utility.
\end{abstract}

Keywords: Characterizing function, decision model, fuzzy data, fuzzy density, fuzzy information

\section{Introduction}

Obtainable information for further decision making often consists of measurement results, for which statistical analysis is in order. The measurement of continuous variables is generally clouded with uncertainty, i.e. data are, more or less, fuzzy. This kind of uncertainty differs from errors. Despite their uncertainties, such data are often essential for decisions and must be carefully analysed for meaningful results. To describe different facets of reality, the analysis methods have to capture this type of uncertainty. Hence, the occurring uncertainty can be modelled using the theory of fuzzy random functions [5] and the related methods, which are available through mathematical models for fuzzy data. Accordingly, application of such methods results in more realistic models for data analysis and, subsequently, better understanding of the collected data for further use of such information in decision making [6].

Methods and models for fuzzy data and their statistical analysis are active fields of research. In these cases, the data to be studied and/or further analyzed are often presented with considerable uncertainties. Nevertheless, such data, despite their uncertainties, are essential for decisions and often critical. Different methods have been developed for analyzing or correcting results from the use of incomplete data [4]. The description of fuzzy data and their statistical analysis also form an active field of research. A modern quantitative description of such data is by special fuzzy sets. The most suitable mathematical model to describe the fuzziness is by means of fuzzy numbers and their characterizing functions [1],[8],[9]. Decision making from fuzzy information takes into account the statistical knowledge of the condition under consideration. In other words, it is the process of fitting a probability (distribution) model to a set of data and summarizing the result by a probability distribution on the parameters of the model and on unobserved quantities [2].

In Section 2, the standard decision model is provided. In Section 3, the mathematical foundations for fuzzy numbers along with their characterizing functions, and fuzzy-valued functions are described. Section 4 introduces the generalized probability distribution to fuzzy densities. In Section 5, a generalized decision model based on fuzzy information is introduced. Finally, Section 6 concludes the paper with final remarks.

\section{The Standard Decision Model}

The formal model for making optimal decisions is defined as following:

$\mathscr{D}=\{$ set of all possible decisions $\mathrm{d}\}$ 
$\Theta=\{$ set of all possible states $\theta$ of the considered system $\}$

$\mathrm{U}(\theta, \mathrm{d})=$ utility of the decision $\mathrm{d}$, if the system is in state $\theta$

$\mathrm{P}=$ probability distribution on the space of states $\Theta$

$$
\mathrm{U}(\theta, \mathrm{d}) \in \mathbb{R} \forall(\theta, \mathrm{d}) \in \Theta \times \mathscr{D}
$$

The optimality criterion for the decision is the expected utility:

$$
\overline{\mathrm{U}}(\mathrm{d}):=\mathbb{E}_{P} \mathrm{U}(\tilde{\theta}, \mathrm{d}),
$$

where $\tilde{\theta}$ is the stochastic quantity describing the state of the system.

In case of finite state space $\Theta=\left\{\theta_{1}, \ldots, \theta_{n}\right\}$ with probabilities $\mathrm{p}\left(\theta_{j}\right)$, we have

$$
\overline{\mathrm{U}}(\mathrm{d})=\sum_{j=1}^{n} \mathrm{U}\left(\theta_{j}, \mathrm{~d}\right) \mathrm{p}\left(\theta_{j}\right) .
$$

For continuous state space $\Theta$ with probability density $f(\cdot)$ on $\Theta$,

is defined.

$$
\overline{\mathrm{U}}(\mathrm{d})=\int_{\Theta} \mathrm{U}(\theta, \mathrm{d}) f(\theta) d \theta
$$

Optimal decisions $\mathrm{d}_{\mathrm{opt}}$ are defined by the following equation:

$$
\mathrm{d}_{\mathrm{opt}}:=\underset{\mathrm{d} \in \mathscr{Q}}{\arg \max } \overline{\mathrm{U}}(\mathrm{d})
$$

Remark 1: In reality, utilities are frequently not precise numbers, and the probability distribution $\mathrm{P}$ is not always known precisely. Therefore, a generalization of the decision model is necessary. This is possible via concepts of fuzzy models.

\section{$3 \quad$ Modeling Fuzziness}

In order to describe fuzzy data, from observations or measurements of continuous quantities, the definition of general fuzzy numbers and fuzzy vectors is useful. In addition, to model fuzzy probability densities, so-called fuzzy-valued functions are necessary.

\subsection{Fuzzy Numbers}

Definition 1: A general fuzzy number $x^{*}$ is defined by its characterizing function $\xi(\cdot)$, which is a real function of one real variable and has the following properties:

(1) $\xi: \mathbb{R} \rightarrow[0,1]$

(2) The support of $\xi(\cdot)$, denoted by $\operatorname{Supp}[\xi(\cdot)]$ and defined by is a bounded subset of $\mathbb{R}$.

$$
\operatorname{Supp}[\xi(\cdot)]:=\{x \in \mathbb{R}: \xi(x)>0\},
$$

(3) For all $\delta \in(0,1]$, the $\delta$-cut $C_{\delta}[\xi(\cdot)]$, defined by

$$
C_{\delta}[\xi(\cdot)]:=\{x \in \mathbb{R}: \xi(x) \geqslant \delta\}=\bigcup_{j=1}^{k_{\delta}}\left[a_{\delta, j}, b_{\delta, j}\right],
$$

is non-empty and finite union of compact intervals.

Definition 2: A fuzzy number whose $\delta$-cuts for all $\delta \in(0,1]$ are compact intervals $\left[a_{\delta}, b_{\delta}\right]$ is called fuzzy interval.

Along with general fuzzy numbers, a related critical question is how to obtain the characterizing function of a measurement result. First, a function has to be defined and then the special membership functions of fuzzy numbers, which are characterizing functions, describing measurement results, can be achieved [7].

The characterizing function $\xi(\cdot)$ can be obtained in the following way:

For example, in case of digital measurement equipment, the result is a "number y" with finitely many digits. The characterizing function $\xi(\cdot)$ is the indicator function $\mathbb{1}_{[\underline{y}, \bar{y}]}(\cdot)$ of the interval $[\underline{y}, \bar{y}]$ where $\underline{y}$ and $\bar{y}$ are the real numbers, obtained by defining the missing digits of y to be " 0 " and "9" respectively.

In case of analog measurement equipment, the measurement result $x$ may be given by a pointer position on a specified scale. This measurement result can then be reported by a photograph. Such photographs are color intensity pictures displaying the color intensity $g(\cdot)$ along a measurable scale. From the color intensity $g(\cdot)$, the characterizing function $\xi(\cdot)$ can be obtained through the function $g(\cdot)$ as defined in the following way:

$$
\xi(x):=\frac{g(x)}{\max \{g(x): x \in \mathbb{R}\}} \forall x \in \mathbb{R}
$$


A measurement $x, \forall x \in \mathbb{R}$, can be given by a color intensity transition $h(\cdot)$. Based on the function $h(\cdot)$, the characterizing function $\xi(\cdot)$ of the fuzzy number describing the measurement is obtained by:

where $h^{\prime}(\cdot)$ is the derivative of the function $h(\cdot)$.

$$
\xi(x):=\frac{\left|h^{\prime}(x)\right|}{\max \left\{\left|h^{\prime}(x)\right|: x \in \mathbb{R}\right\}} \forall x \in \mathbb{R},
$$

\subsection{Fuzzy-Valued Functions}

In order to generalize probability distributions, the concept of densities has to be generalized. This is based on the concept of fuzzy-valued functions.

Definition 3: A function $f^{*}$ defined on a set $M$, which assigns to every $x \in M$, a fuzzy interval $f^{*}(x)$ is called fuzzy function. Denoting the $\delta$-cuts $C_{\delta}\left[f^{*}(x)\right]=\left[a_{\delta}(x), b_{\delta}(x)\right]$, the so-called lower limit and upper limit $\delta$-level functions $f_{\sigma}(\cdot)$ and $\bar{f}_{\sigma}(\cdot)$ are defined in the following way:

Let $C_{\delta}\left[f^{*}(x)\right]=\left[a_{\delta}(x), b_{\delta}(x)\right] \forall \delta \in(0,1]$, the lower limit and upper limit $\delta$-level functions are given by $a_{\delta}(\cdot)$ and $b_{\delta}(\cdot)$, which are standard real-valued functions defined by their values $\underline{f_{\sigma}}(x):=a_{\delta}(x)$ and $\overline{f_{\sigma}}(x):=b_{\delta}(x) \forall x \in M$.

\section{Fuzzy Probability Densities}

A special kind of fuzzy valued functions is fuzzy probability densities.

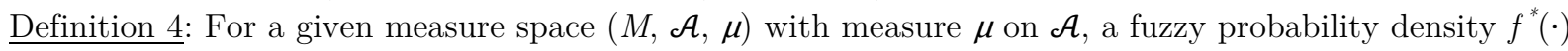
is a fuzzy valued function, whose $\delta$-level functions are all integrable with finite integrals $\int_{M} \bar{f}_{\delta}(x) d \mu(x)$ and $\int_{M} \underline{f}_{\delta}(x) d \mu(x)$, and there exists a standard probability density $g(\cdot)$ on $(M, \mathcal{A})$ obeying $f_{1}(x) \leq g(x)$ $\leq \bar{f}_{1}(x) \bar{\forall} x \in M$.

Based on fuzzy probability densities, generalized so-called fuzzy probabilities of events $A \in \mathcal{A}, P^{*}(A)$ are fuzzy intervals defined in the following way:

The generating family of intervals $A_{\delta}=\left[a_{\delta}, b_{\delta}\right], \delta \in(0,1]$, for the fuzzy interval $P^{*}(A)$ is obtained by

$$
\begin{aligned}
& a_{\delta}:=\inf \left\{\int_{A} h(x) d \mu(x): h \in \mathscr{V}_{\delta}\right\} \\
& b_{\delta}:=\sup \left\{\int_{A} h(x) d \mu(x): h \in \mathscr{D}_{\delta}\right\}
\end{aligned}
$$

where $\mathscr{D}_{\delta}$ is the set of all classical probability densities $h(\cdot)$ on $(M, \mathcal{A})$ obeying

$$
\underline{f_{\sigma}}(x) \leq h(x) \leq \bar{f}_{\sigma}(x) \forall x \in M .
$$

The characterizing function $\xi(\cdot)$ of $P^{*}(A)$ is given by the generation lemma [7]:

$$
\xi(y)=\sup \left\{\delta . \mathbb{1}_{\left[a_{\delta}, b_{\delta}\right]}(x): \delta \in[0,1]\right\} \text { for all } y \in \mathbb{R},
$$

where $\mathbb{1}_{B}(\cdot)$ denotes the indicator function of the set $B$, and $\left[a_{0}, b_{0}\right]:=\mathbb{R}$.

\section{Decisions Based on Fuzzy Information}

In generalizing the concepts from section 2, the following fuzzy concepts are useful:

$\mathrm{U}^{*}(\theta, \mathrm{d})=$ fuzzy interval

$\mathrm{P}^{*}=$ fuzzy probability distribution on $\Theta$

The generalized expected utility of a decision $\mathrm{d}$ is based on the generalized integral

$$
\overline{\overline{\mathrm{U}}}^{*}(d)=\int_{\Theta} \mathrm{U}^{*}(\theta, \mathrm{d}) d P^{*}(\theta)
$$

where this integral is a fuzzy interval which is based on $\delta$-level functions of $\mathrm{U}^{*}(.,$.$) and the fuzzy density$ $f^{*}, \underline{f_{\sigma}}(\cdot), \bar{f}_{\sigma}(\cdot), \overline{\mathrm{U}}_{\delta}(\cdot, \mathrm{d}), \underline{\mathrm{U}}_{\delta}(\cdot, \mathrm{d})$

The generating family for the characterizing function $\varphi_{d}(\cdot)$ of $\overline{\overline{\mathrm{U}}}^{*}(\mathrm{~d})$ are the intervals $\left[a_{\delta}(\mathrm{d}), b_{\delta}(\mathrm{d})\right] ; \delta \in(0,1]$, defined by

$$
\begin{aligned}
a_{\delta} & :=\int_{\Theta} \underline{\mathrm{U}}_{\delta}(\theta, \mathrm{d}) \underline{f_{\sigma}}(\theta) \mathrm{d} \theta \\
b_{\delta} & :=\int_{\ominus} \overline{\mathrm{U}}_{\delta}(\theta, \mathrm{d}) \overline{f_{\sigma}}(\theta) \mathrm{d} \theta .
\end{aligned}
$$

Then $\varphi_{d}(\cdot)$ is given by the generation lemma (also compare with [3]) by its values 


$$
\varphi_{d}(x)=\sup \left\{\delta \cdot \mathbb{1}_{\left[a_{\delta}(\mathrm{d}), b_{\delta}(\mathrm{d})\right]}(x): \delta \in[0,1]\right\} \quad \forall x \in \mathbb{R}
$$

Remark 2: In order to obtain good decisions, it is necessary to compare fuzzy intervals. This is not so simple as comparing real numbers. Optimal decisions should have maximal expected utility.

\section{Final Remark}

Measurement data are essential for making some critical decisions. However, the measurement of continuous variables is often uncertain (differs from error), i.e. many data are more or less fuzzy. Despite their uncertainties, such data are often essential for decisions and have to be carefully analysed for meaningful results. Hence, as the demand for representative models for real observations increases, generalized concepts for quantifying fuzziness are necessary. In this paper, a concept of generalized mathematical model is introduced to encompass the analysis of fuzzy data. The generalized model contains mathematical definitions, which are useful in translating the variability and imprecision of real observations into the models. The theory of fuzzy numbers along with their characterizing functions is applied in capturing the imprecision of continuous quantities. This concept is suitable in modeling fuzzy information, resulting in fuzzy probability densities. The fuzzy probability densities and their $\delta$-level functions are used in defining optimal decision in face of fuzzy information. Altogether, a mathematical model is generalized for modeling fuzzy information with explainable mathematical grounds in capturing the variability and imprecision of real observations.

\section{References}

1. Dubois, D., Prade, H., "Fuzzy Sets and Probability: misunderstandings, bridges, and gaps", Proc. of the $2^{\text {nd }}$ IEEE International Conference of Fuzzy Systems, IEEE, Piscataway, 1993, pp. 1059-1068.

2. Gelman, A., Carlin, J., Stern, H., Dunson, D., Vehtari, A., and Rubin, D., Bayesian Data Analysis, $3^{\text {rd }}$ Edition, Boca Raton, Florida: Chapman \& Hall/CRC, 2014

3. Kovářová, L., Viertl, R., "The Generation of Fuzzy Sets and the Construction of Characterizing Functions of Fuzzy Data", Iranian Journal of Fuzzy Systems 12(6), 2015, pp. 1-16.

4. Krasker, W. S. et al., Chapter 11: Estimation for Dirty Data and Flawed Models, Handbook of Econometrics 1, 1983, pp. 651-698.

5. Möller, B., and Reuter, U., Uncertainty Forecasting in Engineering, Berlin: Springer-Verlag, 2007.

6. Sunanta, O., Viertl, R., "Fuzziness and statistics - mathematical models for uncertainty", Theoretical and Practical Research in Economic Field, (Volume VIII, Summer 2017), 1(15): 2017, pp. 31-46.

7. Viertl, R., Statistical Methods for Fuzzy Data, Chichester: Wiley, 2011.

8. Viertl, R., "Measurement of Continuous Quantities and their Statistical Evaluation", Austrian Journal of Statistics 44, 2015, pp. 25-32.

9. Zadeh, L. A., "Discussion: Probability Theory and Fuzzy Logic Are Complementary Rather Than Competitive", Technometrics 37(3), 1995, pp. 271-276. 